\title{
Over 300-km Transmission of 25 Gb/s Optical SSB NPAM-4 Signal with Electronic Dispersion Pre-compensation and Interference Mitigation
}

\author{
Mingyue Zhu ${ }^{(1)}$, Jing Zhang ${ }^{(1, *)}$, Xingwen Yi( ${ }^{(1)}$, Hao Ying ${ }^{(1)}$, Zengjie Zhang ${ }^{(1)}$, Feng Wen ${ }^{(1,2)}$, Longjiang Li ${ }^{(3)}$, \\ Mian Zheng ${ }^{(4)}$, and Kun Qiu ${ }^{(1)}$ \\ ${ }^{(1)}$ Key Laboratory of Optical Fiber Sensing and Communications, University of Electronic Science and Technology of China, Chengdu 611731, \\ China \\ ${ }^{(2)}$ Aston Institute of Photonic Technologies, Aston University, B4 7ET Birmingham, UK \\ ${ }^{(3)}$ Unit 78111 of Chinese People's Liberation Army, Sichuan, 610031, China \\ ${ }^{(4)}$ School of Electrical Engineering and Information, Southwest Petroleum University, Chengdu 610500, China \\ zhangjing1983@uestc.edu.cn
}

\begin{abstract}
We experimentally demonstrate the interference mitigation in direct-detection of optical SSB signals with Nyquist-PAM-4. At $25 \mathrm{~Gb} / \mathrm{s}$, we achieve over 300-km and 500-km SSMF with an average BER of $2.7 \times 10^{-3}(<\mathrm{HD}-\mathrm{FEC})$ and $9.4 \times 10^{-3}$ ( $\left.<\mathrm{SD}-\mathrm{FEC}\right)$, respectively.

OCIS codes: (060.0060) Fiber optics and optical communications; (060.2330) Fiber optics communications; (060.4080) Modulation
\end{abstract}

\section{Introduction}

There is an increasing demand of deploying the high-speed and low-cost optical communications over distances of up to $300 \mathrm{~km}$ for inter-data-centre interconnection and metro networks [1]. Despite the superior performance of coherent detection, intensity modulation and direct-detection (IMDD) is still preferred for short- and mediumdistance optical communications due to its low cost, small size and low power dissipation. Recently, advanced modulation formats such as discrete multi-tone, carrier-less amplitude and phase modulation and pulse-amplitude modulation (PAM) are used to achieve high spectral efficiency (SE) and reduce the cost per bit [2]. In general, PAM-4 has a simpler architecture and low power consumption, which are favourable in short-distance optical communications [3]. In conventional IMDD systems, the optical carrier is modulated to generate a double sidebands (DSB) signal. The two sidebands experience different phase shifts and introduce self-cancellation at certain frequencies, depending upon the accumulated chromatic dispersion (CD). Optical single side-band (SSB) modulation has been proposed to alleviate the power fading effect and double the spectra efficiency. Generally, two main methods, complex modulation with a pair of Hilbert transform signals and vestigial sideband filtering (VSB), have been proposed to generate a SSB signal $[3,4]$. All of these works concentrate on the improvement of CD tolerance in the C-band, which makes full compensation of the CD desirable [1]. Moreover, considering the cost and a high roll-off factor requirement of the optical filter, complex modulation with a pair of Hilbert transform signals is preferred to generate the SSB signal and it also enables electrical dispersion pre-compensation (EDC) at the transmitter side. Although SSB can avoid the power fading, both the Hilbert transform term of the baseband transmitted signal and signal-signal beat interference (SSBI) are detected after direct-detection, which introduce the linear and nonlinear interference, respectively.

In this paper, a dual-drive Mach-Zehnder modulator (DDMZM) has been employed to realize complex modulation with a pair of Hilbert transform signals to realize the optical SSB modulation. We first study two linear interference cancellation schemes in an optical SSB Nyquist-pulse shaping PAM-4 (NPAM-4) system. Then, the EDC and a Hybrid equalizer which combines feed-forward equalizer (FFE) and higher order Volterra terms is used to improve the CD tolerance and mitigate the SSBI. Subsequently, $25 \mathrm{~Gb} / \mathrm{s}$ NPAM-4 signal with a high SE up to $3.96 \mathrm{bit} / \mathrm{s} / \mathrm{Hz}$ is successfully transmitted over $300 \mathrm{~km}$ and $500 \mathrm{~km} \mathrm{SSMF}$, respectively.

\section{Principle}

The DDMZM can be modelled as two phase modulators in parallel. To generate the optical SSB signal, the baseband NPAM-4 signal $m(t)$ and its Hilbert transform $\hat{m}(t)$ are used to drive the DDMZM which is biased at the quadrature point. When the driving signals are small, the output of DDMZM can be approximated as,

$$
E_{\text {out }}(t) \approx \frac{E_{i n}(t)}{2}\left\lfloor 1-j+\frac{\pi}{V_{\pi}}(m(t)+j \hat{m}(t))\right\rfloor
$$

where $E_{i n}(t)$ is the input optical field and $V_{\pi}$ is the half-wave voltage. After the square-law detection and DC blocking, the received signal is, 


$$
r(t)=\left|E_{\text {out }}(t)\right|^{2}=\frac{\pi\left|E_{\text {in }}(t)\right|^{2}}{2 V_{\pi}}[m(t)-\hat{m}(t)]+\frac{\pi^{2}\left|E_{\text {in }}(t)\right|^{2}}{4 V_{\pi}^{2}}\left[m^{2}(t)+\hat{m}^{2}(t)\right]
$$

In Eq. (2), in addition to the transmitted baseband signal, its Hilbert transform $\hat{m}(t)$ and SSBI are also detected, which introduce the $1^{\text {st }}$-order and $2^{\text {nd }}$-order interference, respectively. As $V_{\pi}$ of DDMZM is normally larger than $\pi$, the $1^{\text {st }}$-order interference in Eq. (2) is relative large. In order to eliminate the $1^{\text {st }}$-order interference, we utilize the characteristic of the Hilbert transform at the receiver and the transformation of the baseband signals at the transmitter to cancel the $1^{\text {st }}$-order interference, respectively. First, we apply the Hilbert superposition (HS) at the receiver, denoted as Case-I. Eq. (2) can be rewritten as,

$$
\tilde{r}(t)=r(t)+H[r(t)]=2 A m(t)+B\left\{m^{2}(t)+\hat{m}^{2}(t)+H\left[m^{2}(t)+\hat{m}^{2}(t)\right]\right\}
$$

where, $H[\bullet]$ means the Hilbert transform, $A$ and $\mathrm{B}$ are proportional to $\pi\left|E_{i n}(t)\right|^{2} /\left(2 V_{\pi}\right)$ and $\pi^{2}\left|E_{i n}(t)\right|^{2} /\left(4 V_{\pi}\right)^{2}$, respectively. In Eq. (3), we find that the $1^{\text {st }}$-order interference from the Hilbert transform of the baseband signal is eliminated after HS.

Other than the mitigation scheme at the receiver, we also find that we can substitute the original signal by the sum and difference signal in Eq. (2), i.e., $m(t)+\hat{m}(t)$ and $\hat{m}(t)-m(t)$, to remove $1^{\text {st }}$-order interference, denoted as Case-II. Note that they are also a pair of Hilbert signals. Thus, the Eq. (1) can be expressed as,

$$
E_{\text {out }}(t)=\frac{E_{\text {in }}(t)}{2}\left\lfloor 1-j+\frac{\pi}{V_{\pi}}(m(t)+\hat{m}(t))+j \frac{\pi}{V_{\pi}}(\hat{m}(t)-m(t))\right\rfloor
$$

After the square-law detection and DC blocking, the received signal can be written as,

$$
r(t)=2 A m(t)+B\left(m^{2}(t)+\hat{m}^{2}(t)\right)
$$

We find that in Case-II: (i) the sum and difference input signals are also a pair of Hilbert transform signals and the character of SSB is not changed; (ii) the Hilbert transform of the original baseband signal is eliminated in Eq. (4).

\section{Experimental Setup and Results}
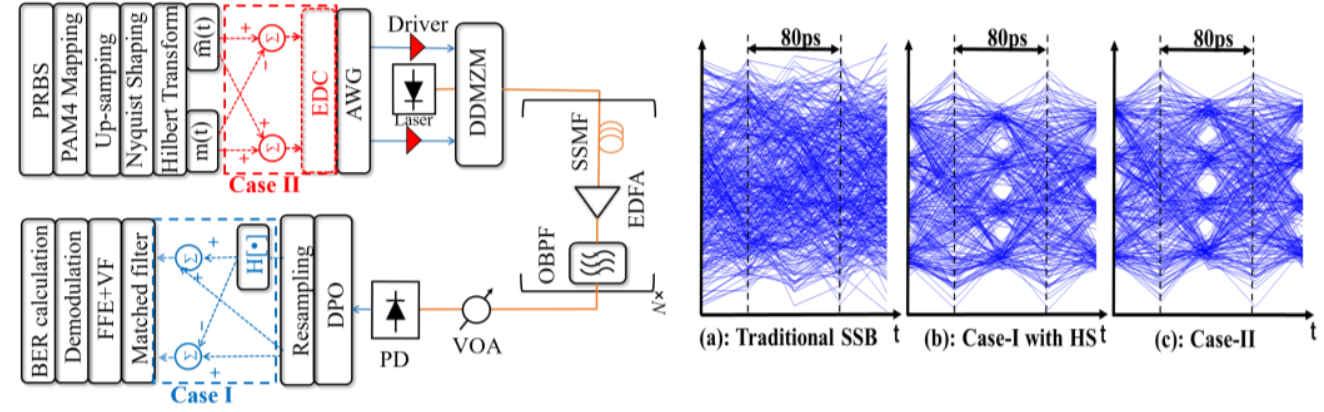

Fig. 1. Experimental schematic, VF: Volterra filter

Fig. 2. The eye diagrams of the received signal

The experimental configuration is illustrated in Fig. 1. At the transmitter, a pseudo random bit sequence (PRBS) of $2^{18}$ bits is generated for PAM-4 modulation and up-sampled to 2 sample-per-symbol. The Nyquist filter with a roll-off factor of 0.01 is applied to generate NPAM-4 sequence and its Hilbert term is generated by Hilbert transform. In Case-I, we use the baseband signal $m(t)$ and its Hilbert term as the driving signals for DDMZM, which has no change with a conventional optical SSB transmitter [4]. In Case-II, the sum and difference of the baseband NPAM-4 signals are used as the driving signals. The driving signals are generated by an AWG700002A at its highest sample rate of $25 \mathrm{GS} / \mathrm{s}$. Hence, the signal bit rate is $25 \mathrm{~Gb} / \mathrm{s}$. A DDMZM with $V_{\pi}$ at $3.8 \mathrm{~V}$ is biased at the quadrature point to generate the optical SSB signal, which enables the CD pre-compensated. For the DD system, the carrier to signal power ratio (CSPR) is an important parameter to characterize the system. The optimized CSPR is mainly limited by the nonlinearity of DDMZM [2], therefore, we adjust the optical modulation index (OMI) by changing the amplitude of the driving signals for optimal CSPR, where $O M I=\left(V_{R F}\right)_{R M S} / V_{\pi}$, and $\left(V_{R F}\right)_{R M S}$ is the root-mean-square (RMS) amplitude of the electrical signals. According to the numerical calculation and experimental test, the OMI is set at 0.13. An optical source with $12 \mathrm{dBm}$ output optical power is fed into the DDMZM, and the output optical signal with $0 \mathrm{dBm}$ launch power transmits over the SSMF. The transmission link is comprised $N$ spans of $100 \mathrm{~km} \mathrm{SSMF}$ with an attenuation coefficient of $0.2 \mathrm{~dB} / \mathrm{km}$ and an average CD coefficient of $16.8 \mathrm{ps} /(\mathrm{nm} \cdot \mathrm{km})$. The fiber loss is compensated by an Erbium-doped fiber amplifier (EDFA) and an optical band-pass filter (OBPF) is used to suppress the noise. A variable optical attenuator (VOA) is employed to adjust the received optical power (ROP). As we use the Nyquist pulse shaping, the resulted narrow spectrum also help us to use $10 \mathrm{GHz}$ devices for $25 \mathrm{~Gb} / \mathrm{s}$ transmission. At the receiver, the optical signal is detected by a commercial photodiode (PD) with $10 \mathrm{GHz}$ bandwidth and sampled by a DPO72504D operating at a sampling rate of $50 \mathrm{GS} / \mathrm{s}$. Then, the received electrical 
signal is processed in offline DSP. A Hybrid equalizer which combines FFE and higher order Volterra terms is used to shorten the channel impulse response and mitigate the SSBI [5]. We compare the transmission performance of the traditional SSB scheme, Case-I and Case-II, respectively. At B2B case, the eye diagrams of NPAM-4 without any equalization signal for the three schemes are shown in Fig. 2. As shown in Fig. 2 (a), the eye diagram is closed in conventional optical SSB scheme due to the $1^{\text {st }}$-order interference, where the Hilbert transform can be eliminated by mixing with a RF local oscillator and filtered by a low-pass filter in an IF-based system. In Fig. 2 (b) and (c), the eye diagrams are clearly open for Case-I and Case-II. This is because the $1^{\text {st }}$-order interference has been eliminated.

Based on the eye diagrams, we find that the received signal cannot be directly demodulated without linear equalization in conventional SSB system at B2B case. Therefore, we use LMS-based FFE with 11 taps to recover the received signals. Fig. 3 depicts the experimental BER performance with FFE at different ROPs. As shown in Fig. (3), the BER performance is slightly better for Case-II, which is because the interference is directly cancelled after PD in Eq. (5). While the $1^{\text {st }}$-order interference cancellation is operated at the receiver by HS for Case-I in Eq. (3), its effect depends on the received signal and errors of the received signal will limit the effectiveness of HS.

We use the two schemes to transmit the $25 \mathrm{~Gb} / \mathrm{s}$ NPAM-4 signal over the fiber link. The dispersion is a main limiting factor as the transmission distance increases in direct-detection systems. The optical SSB signal is generated by DDMZM with complex modulation in our experiment, which enables digital EDC due to the complex field modulation characteristic. A Hybrid equalizer is applied to mitigate the SSBI. The kernels and memory length of higher-order Volterra terms are determined by the Recursive Least Square algorithm. Fig. 4 shows the transmission performance after $300 \mathrm{~km} \mathrm{SSMF} \mathrm{transmission.} \mathrm{The} \mathrm{memory} \mathrm{lengths} \mathrm{of} \mathrm{the} \mathrm{Hybrid} \mathrm{equalizer} \mathrm{are} \mathrm{set} \mathrm{at}(51,21,5)$. As shown in Fig. 4, the performance without EDC for Case-II is slightly better than that for Case-I, while the BERs for the two schemes are in the same order of magnitude and noise is no longer the main limitation as the B2B case. The two schemes have similar transmission performance and they both can be below the 7\% HD-FEC threshold with EDC, which is because the pre-distorted signal is transmitted and residual interference can be mitigated by the Hybrid equalizer. We extend the fiber distance and show the BER performance at different fiber lengths in Fig. 5. Case I and Case II perform similarly in Fig. 5. The transmission performance is improved with EDC and the average BERs are $2.7 \times 10^{-3}\left(<\right.$ HD-FEC) and $9.4 \times 10^{-3}(<$ SD-FEC) after $300 \mathrm{~km}$ and $500 \mathrm{~km}$ fiber transmission.

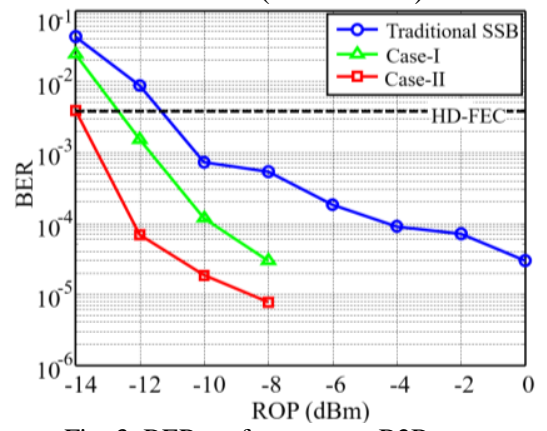

Fig. 3. BER performance at B2B

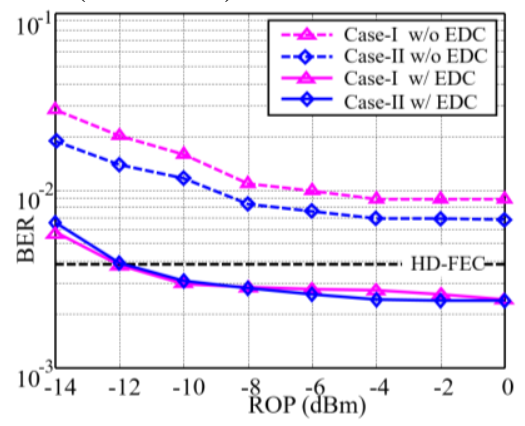

Fig. 4. BER performance over $300 \mathrm{~km} \mathrm{SSMF}$

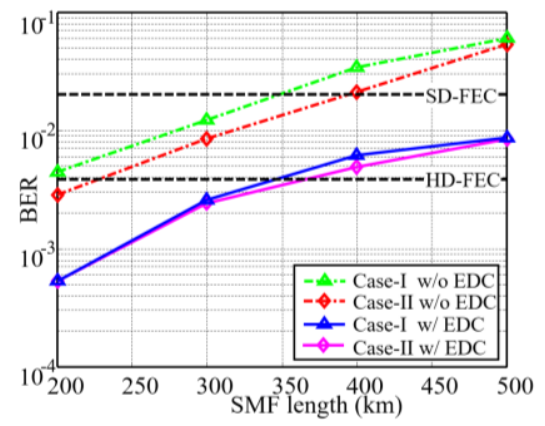

Fig. 5. BER versus fiber lengths

\section{Conclusions}

We have experimentally demonstrated two linear interference cancellation schemes in direct detection of optical SSB NPAM-4 signals. Based on the two schemes, we have successfully transmitted $25 \mathrm{~Gb} / \mathrm{s}$ Nyquist-PAM-4 signal over $300 \mathrm{~km}$ and $500 \mathrm{~km}$ SSMF.

\section{Acknowledgements}

This work was supported in part by National High Technology Research and Development Program of China (863 Program) (2015AA015501), NSFC (No. 61405024, No. 61420106011 and No.61471088) and project of education department of Sichuan Province (15ZB0063).

\section{References}

[1] Z. Liu, et al., "300-km Transmission of Dispersion Pre-compensated PAM4 Using Direct Modulation and Direct Detection," Optical Fiber Communication. 2017, pp. Th3D.6.

[2] M.Y. Zhu, et al., "Hilbert superposition and modified signal-to-signal beating interference cancellation for single side-band optical NPAM-4 direct-detection system," Optics Express 25(11), 2017, pp. 12622-12631.

[3] J. Lee, et al., "112-Gbit/s Intensity-Modulated Direct-Detect Vestigial Sideband PAM4 Transmission over an 80-km SSMF Link," European Conference and Exhibition on Optical Communication. 2016, pp. M.2.D.3.

[4] L. Zhang, et al., "C-band Single Wavelength 100-Gb/s IM-DD Transmission over 80-km SMF without CD compensation using SSB-DMT," Optical Fiber Communication. 2015, pp. Th4A.2.

[5] J. Zhang, et al., "An Efficient Hybrid Equalizer for $50 \mathrm{~Gb} / \mathrm{s}$ PAM-4 Signal Transmission Over $50 \mathrm{~km}$ SSMF in a 10-GHz DML-Based IM/DD system," Conference on Lasers and Electro-Optics. 2017, pp. SF1L.1. 